Internat. J. Math. \& Math. Sci.

Vol. 24, No. 1 (2000) 63-65

S0161171200003367

(c) Hindawi Publishing Corp.

\title{
SOLVING LINEAR DIFFERENTIAL EQUATIONS BY QUADRATURES: COMMENTS ON A GENERAL PROCEDURE
}

\author{
G. CAVIGLIA and A. MORRO
}

(Received 3 May 1999)

\begin{abstract}
A procedure is examined which solves systems of linear differential equations by quadratures. A direct check shows that a necessary condition for the procedure cannot be true and hence the procedure does not work.
\end{abstract}

Keywords and phrases. Variation of constants technique, solution by quadratures, ordinary linear differential equations.

2000 Mathematics Subject Classification. Primary 34A05, 34A30.

1. Introduction. A procedure set up in [2] provides the solution by quadratures of any non-autonomous linear system of ordinary differential equations. The validity of this procedure would be an outstanding result. With this motivation, we have examined the technical aspects of the related proofs. As a consequence of our investigation we have ascertained that the procedure is based on a hypothesis which cannot be true and hence that the procedure does not work.

In this paper, we review briefly the main steps of the procedure and then show why it cannot work. It then follows that the possibility of solving by quadratures a non-autonomous linear system has still to be proved.

2. The auxiliary system. Following the notation of [2], we denote by capital letters $n \times n$ matrices whose entries are continuous functions of the independent variable $x \in \mathbb{R}$ and we use a prime to mean the derivative with respect to $x$. We consider the matrix form of a linear homogeneous system

$$
Y^{\prime}=F Y
$$

where $F$ is a given invertible matrix and $Y$ is the unknown matrix. It is not restrictive to assume that the system is homogeneous, in that the general solution of nonhomogeneous systems can be obtained by the variation of constants formula [1], i.e., by quadratures, from a fundamental matrix solution of the associated homogeneous system.

It is easily seen that if $F$ is a lower triangular matrix then $n$ independent columns of $Y$ are obtained by quadratures. According to [2], if $F$ is not diagonal then an auxiliary system

$$
X^{\prime}=T X-S
$$


can be constructed such that: (i) $T$ is lower triangular and hence $X$ is ultimately determined by quadratures; (ii) any matrix solution to (2.2) is also a solution to (2.1). Indeed, if $T$ and $S$ are properly defined then $F X=T X-S$, for any non-vanishing $X$, whence it follows that (2.1) holds with $Y$ replaced by $X$, if $X$ solves (2.2).

The auxiliary system (2.2) is obtained by the following procedure. Consider any matrices $A, T, C$, such that

- $T$ is lower triangular,

- $A,(F A-I),(T A-I)$, are non-singular,

where $I$ denotes the identity matrix. Introduce the matrix $P$ defined by

$$
P=(F A-I)(T A-I)^{-1},
$$

and suppose that the matrix $[(I-P)-(F-P T) C]$ is non-singular. Next define $B, U$, and $S$ through

$$
B=[(I-P)-(F-P T) C]^{-1}(F-P T) A, \quad U=A+C B, \quad S=P^{-1}(I-P) U .
$$

The matrices $U-A B$ and $T U-B$ are assumed to be non-singular and this is essential for the procedure to hold.

Next any non-vanishing matrix $X$ is claimed to be representable as

$$
X=A W+U V,
$$

where $W$ and $V$ are given by

$$
\begin{gathered}
A W=X-U(U-A B)^{-1}[X-A T X+A S+A U], \\
V=(U-A B)^{-1}(X-A T X+A S+A U) .
\end{gathered}
$$

On the basis of (2.5), (2.6) it is shown that $F X-T X-S=0$ whence $X=A W+U V$ is a solution to (2.2). This in turn would show that (2.1) can always be solved by quadratures.

3. Failure of the proof. As a consequence of (2.3) and (2.4) the matrix $U-A B$ vanishes identically. To prove that this is so we first observe that, by the definition of $P$,

$$
I-P=(F-P T) A .
$$

Substitution into the definition of $B$ provides

$$
B=[(F-P T) A-(F-P T) C]^{-1}(F-P T) A=[A-C]^{-1} A .
$$

Finally, comparison with (3.2) and the definition of $U$ yields

$$
U-A B=A+(C-A)(A-C)^{-1} A \equiv 0 .
$$

Hence $U-A B$ is not invertible and the proof of (2.2) fails. 


\section{REFERENCES}

[1] J. Kurzweil, Ordinary differential equations, Introduction to the Theory of Ordinary Differential Equations in the Real Domain. Translated from Czech by Michal Basch, Elsevier Scientific Publishing Co., Amsterdam, 1986, p. 440. MR 88m:34001. Zbl 667.34002.

[2] L. K. Williams, Linear and Riccati matrix equations, Internat. J. Math. Math. Sci. 12 (1989), no. 1, 131-136. MR 89k:34002. Zbl 672.34001.

G. Caviglia: Department of Mathematics, Via Dodecaneso 35, 1646 Genova, Italy

A. Morro: University of GenoA, Dibe, Via Opera Pia 11A, 16145 Genova, ItAly

E-mail address: morro@dibe.unige.it 


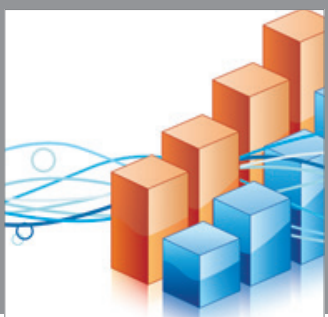

Advances in

Operations Research

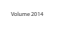

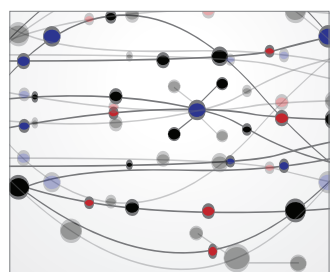

\section{The Scientific} World Journal
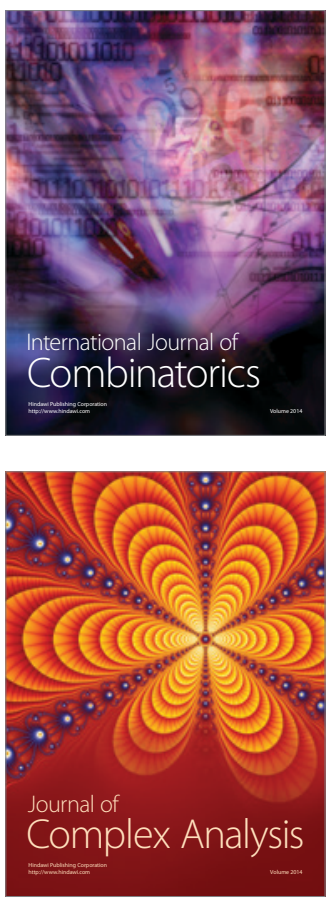

International Journal of

Mathematics and

Mathematical

Sciences
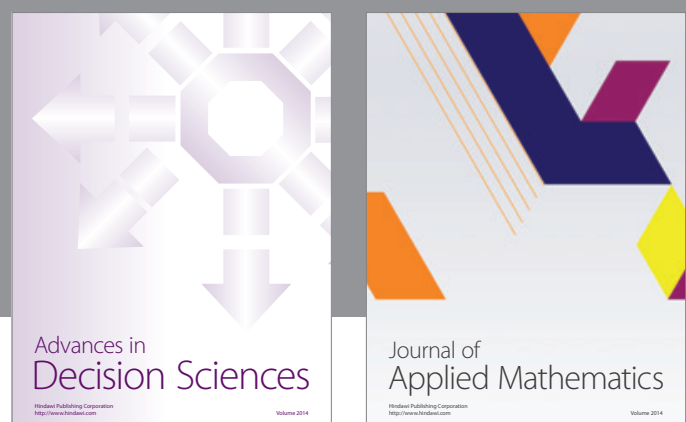

Journal of

Applied Mathematics
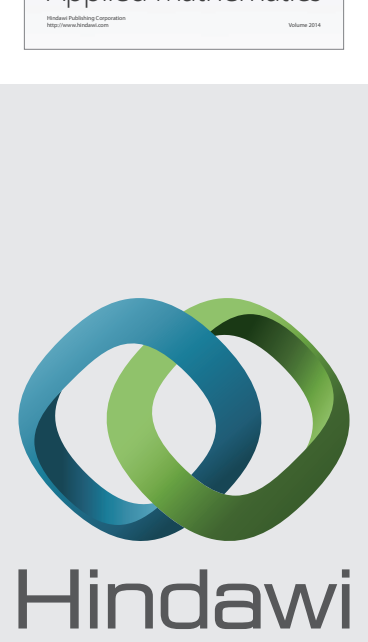

Submit your manuscripts at http://www.hindawi.com
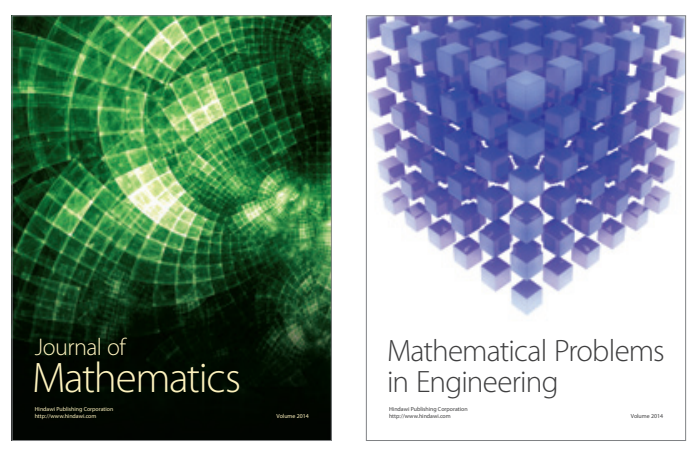

Mathematical Problems in Engineering
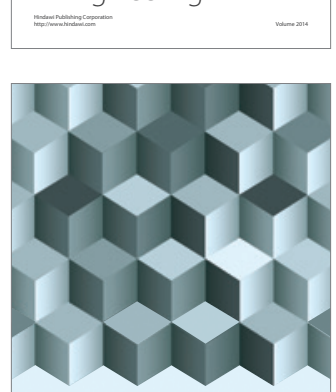

Journal of

Function Spaces
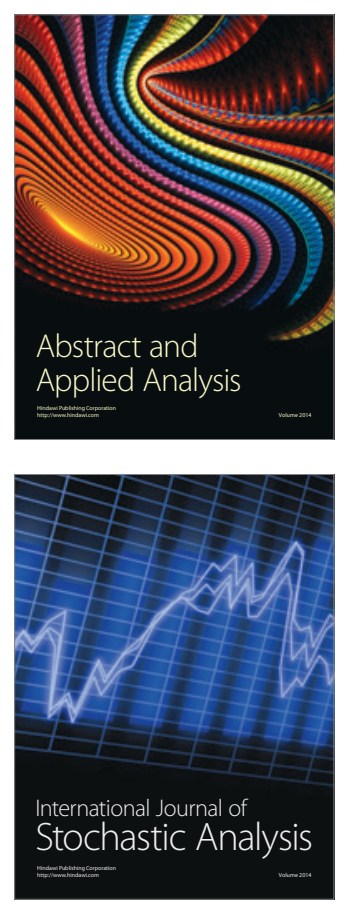

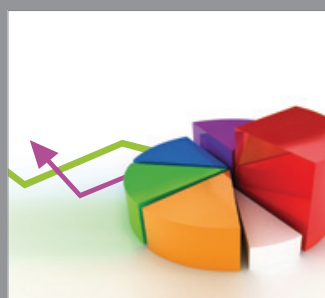

ournal of

Probability and Statistics

Promensencen
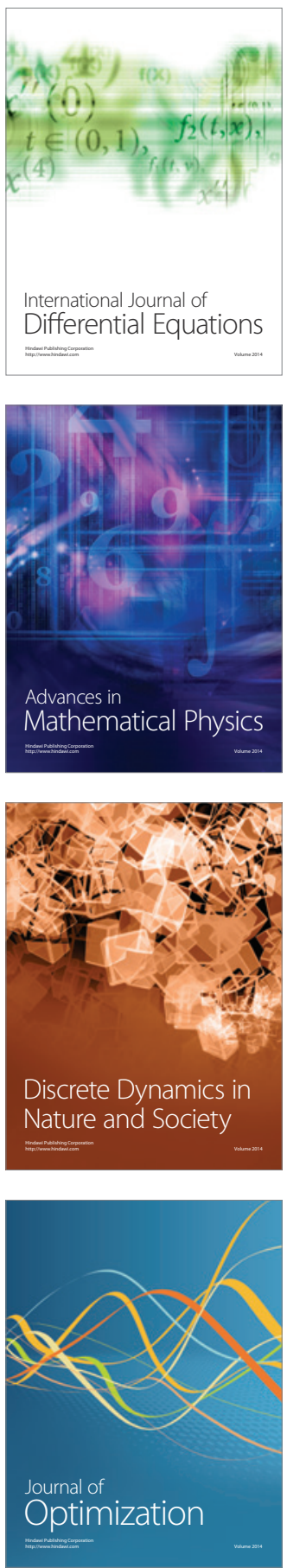